\title{
Examination of drivers' cell phone use behavior at intersections by using naturalistic driving data
}

Huimin Xiong, Shan Bao, Kazuma Kato, James Sayer

$$
8 / 26 / 2014
$$

$4^{\text {th }}$ Naturalistic Driving Research Symposium Blacksburg, VA 


\section{Introduction}

- Driving simulator studies of cell phone-related distraction

- Driving performance decreased and crash risk assumed to increase (Drews, et al., 2009; Liang \& Lee, 2010)

- Decrements in lane-keeping, increases in speed variability (Crisler, et al., 2008)

- Increases in following distance variability (Hosking, et al., 2009; Owens, et al., 2011)

- Survey study of cell phones and safety

- Cell phone use steadily increased while crash rates declined during the same time period (Insurance Institute for Highway Safety, 2010). 


\section{Research Question}

- How driver behave when they use cellphone

- Naturalistic driving environment

- Specific scenario: go through signalized intersections

- Driving performance: speed

- Situational factors: lighting conditions, traffic conditions 


\section{Data Resources: IVBSS}

- Naturalistic driving data from Integrated vehicle based safety system (IVBSS) program

- 5-year long program

- Integrated four types of warnings FCW, LDW, LCM, and CSW

- 16 instrumented research vehicles (2006 Honda Accord)

- 108 drivers (6 weeks of driving for each)

- Younger drivers ( $M=25.2 ; S D=2.9$ )

- Middle-aged drivers ( $M=46.0 ; \mathrm{SD}=3.0$ )

- Older drivers ( $M=64.6 ; \mathrm{SD}=2.8$ ) 


\section{IVBSS: Instrumented vehicles}
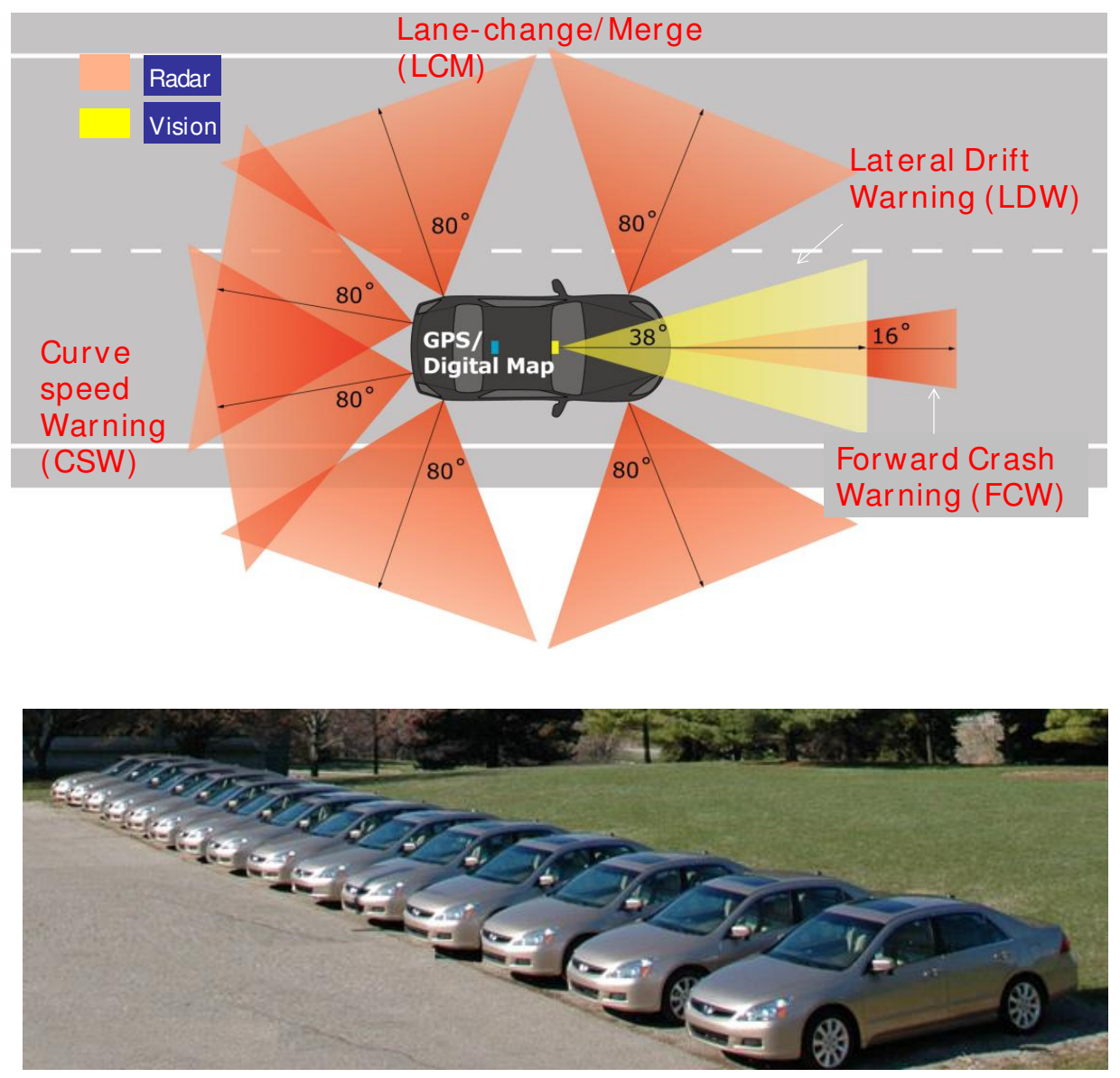


\section{IVBSS: Data Collection}

- April 2009 to April 2010

- Data from 108 drivers

- Data Set

- Over 213K miles

- 23K trips

- 6,200 hours

- 600 data channels

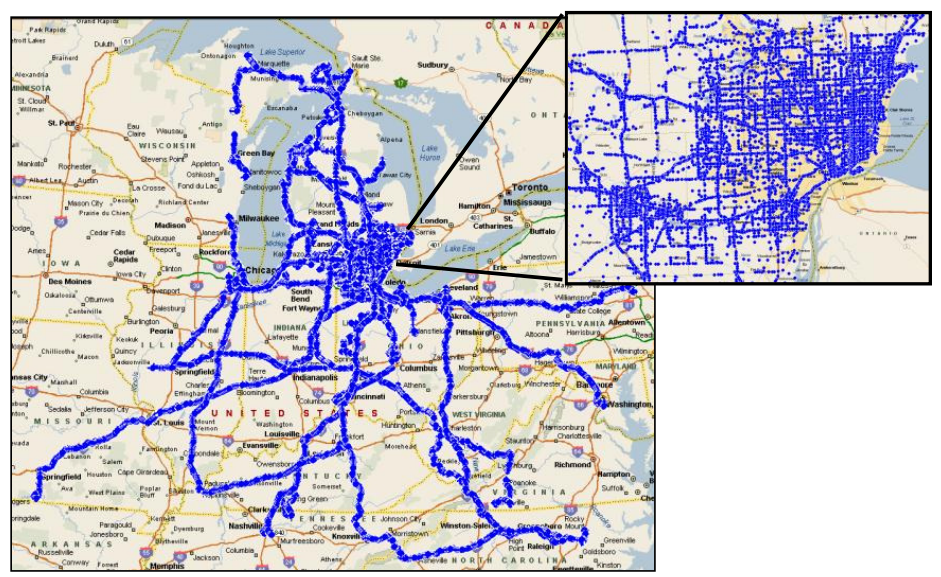

- 5 video channels 


\section{IVBSS: Video Data}

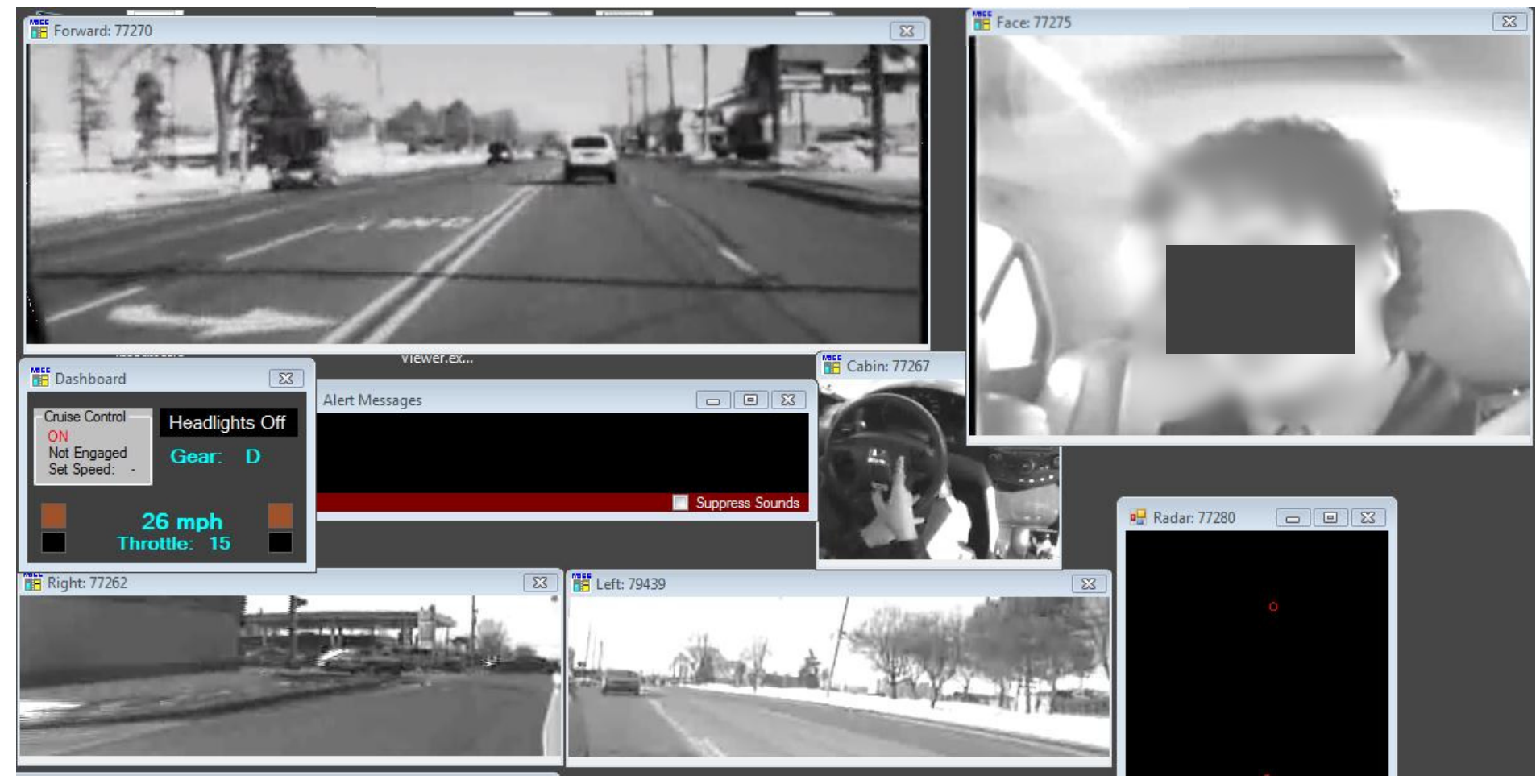

Trained coders went through one week video data, 1381 conversations, 2149 Visual/Manual (VM) tasks 


\section{Method}

- Case Control Study

- Case: went through signalized intersections

- Control: match on the same driver and intersection

- One way ANOVA

- Test average, maximum and minimum driving speed between cell phone use and baseline (driving only)

- Mixed model

- Dependent variable: average speed

- Explanatory variables: traffic condition and lighting condition (situational factors), cellphone use condition 


\section{5 s Samples for Going Through Signalized Intersections}

- Signalized intersections were identified based on HPMS data base

Cell phone use

- Baselines were matched based on driver and intersection

- Min speed over $15 \mathrm{~s}$ (10s before the intersection $+5 \mathrm{~s}$ after) $>$ $8.9 \mathrm{~m} / \mathrm{s}=20 \mathrm{mph}$

- Conversation (453), baseline (647)

- VM (141), baseline (149)

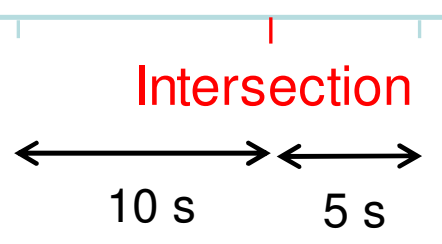




\section{Results: Conversation}

- ANOVA for conversation and baseline

- Significant differences on max and mean speed

- No significant differences on minimum speed

- The differences were small (but over 15 seconds of driving)

Speed Difference $\mathrm{m} / \mathrm{s}(\mathrm{mph}) \quad \mathrm{p}$-value

\begin{tabular}{rrr} 
Maximum & $0.67(1.50)$ & $<0.001$ \\
Mean & $0.43(0.96)$ & 0.05 \\
Minimum & $0.22(0.49)$ & 0.35 \\
\hline
\end{tabular}




\section{Results: Conversation (Cont.)}

- Mixed model showed the consistent results

- Drove slower with conversation

- Lighting situation not significant

- Significant interaction between traffic and conversation

\begin{tabular}{lrrr|}
\hline Factors & Estimates & SE & p-value \\
\hline Intercept & 18.33 & 0.38 & $<0.01$ \\
\hline Conversation & -0.67 & 0.24 & $<0.01$ \\
\hline Moderate traffic & -0.74 & 0.24 & $<0.01$ \\
Dense traffic & -0.53 & 0.33 & n.s. \\
\hline Conversation : Moderate traffic & 0.11 & 0.31 & n.s. \\
Conversation : Dense traffic & 1.12 & 0.54 & 0.04 \\
\hline
\end{tabular}




\section{Results: Conversation (Cont.)}

- ANOVA for each traffic condition

- Sparse and moderate had similar pattern, lower speed with conversation compared with baseline

- Relative balanced sample size

- Sparse: $\Delta_{\mathrm{B}-\mathrm{C}}=0.43 \mathrm{~m} / \mathrm{s}, \mathrm{p}=0.02$

- Moderate: $\Delta_{\mathrm{B}-\mathrm{C}}=0.67 \mathrm{~m} / \mathrm{s}, \mathrm{p}=0.005$

- Dense had opposite pattern, higher speed with conversation

- $\Delta_{\mathrm{B}-\mathrm{C}}=-0.75 \mathrm{~m} / \mathrm{s}, \mathrm{p}=0.43$

- 47 conversation (10\%), 107 baseline 


\section{Results: VM Tasks}

- ANOVA for VM tasks and baseline

- Significant differences on max and mean speed

- No significant differences on minimum speed

- The differences were greater

\begin{tabular}{rrr}
\hline Speed Difference m/s $(\mathrm{mph})$ & $\mathrm{p}$-value \\
\hline Maximum & $1.36(3.04)$ & 0.005 \\
Mean & $1.24(2.77)$ & 0.004 \\
Minimum & $0.84(1.88)$ & 0.9 \\
\hline
\end{tabular}




\section{Results: VM Tasks (Cont.)}

- Mixed model showed the consistent results

- Drove slower with VM tasks

- Lighting situation not significant

- Significant interaction between traffic and VM tasks

\begin{tabular}{lrrr}
\hline Factors & Estimates & SE & p-value \\
\hline Intercept & 18.97 & 0.58 & $<0.01$ \\
\hline VM tasks & -1.76 & 0.49 & $<0.01$ \\
\hline Moderate traffic & 0.27 & 0.57 & n.s. \\
Dense traffic & -0.72 & 0.80 & n.s. \\
VM: Moderate traffic & 0.79 & 0.79 & n.s. \\
VM: Dense traffic & 2.54 & 1.08 & 0.02 \\
\hline
\end{tabular}




\section{Results: VM Tasks (Cont.)}

- Sparse and moderate: lower speed with VM tasks

- Sparse: $\Delta_{\mathrm{B}-\mathrm{T}}=2.15 \mathrm{~m} / \mathrm{s}, \mathrm{p}<0.01$

- Moderate: $\Delta_{\mathrm{B}-\mathrm{T}}=0.53 \mathrm{~m} / \mathrm{s}, \mathrm{p}=0.1$

- Dense: higher mean speed with VM tasks, $\Delta_{\mathrm{B}-\mathrm{T}}=-$ $0.74 \mathrm{~m} / \mathrm{s}, \mathrm{p}=0.46,18 \mathrm{VM}$

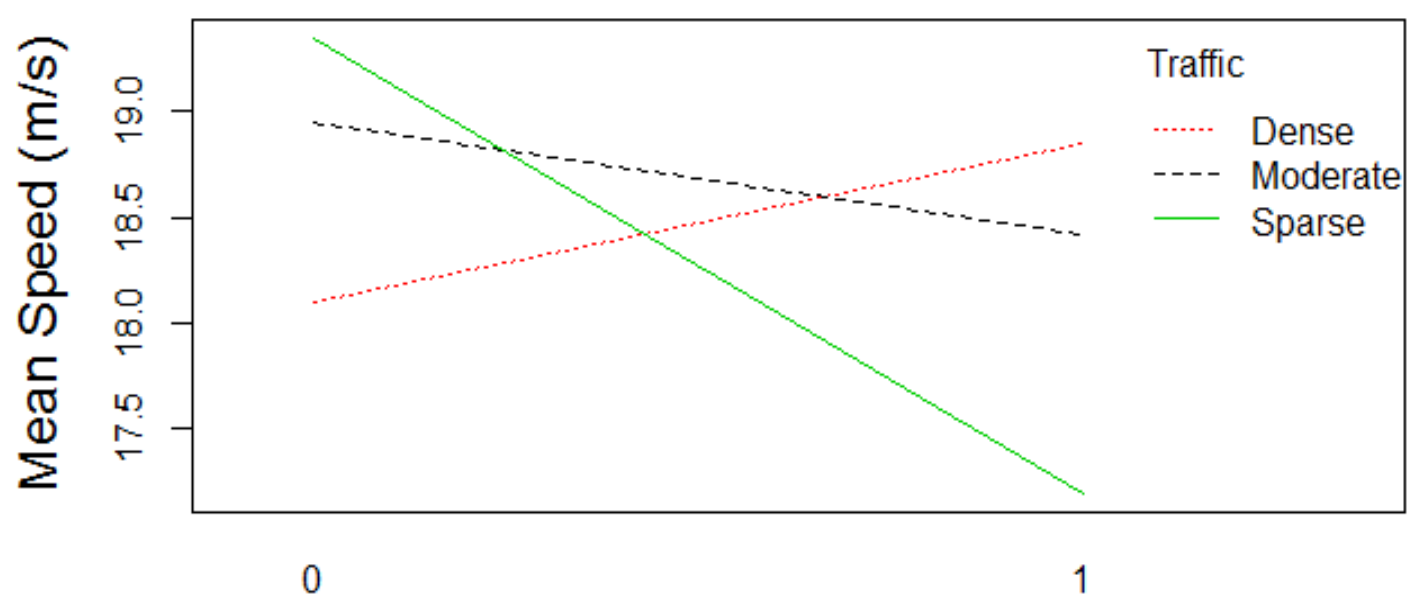

Cell phone VM tasks 


\section{Discussion}

- Adaptive behavior

- Reduced speed more with VM vs Conversations

- Adapt their behaviors to compensate for higher driving demand

- Significantly much lower speed with VM tasks under sparse traffic

- Drivers engage in VM tasks in low demand situations (sparse traffic)

- Reduced speed as well to compensate for the increased demand from secondary tasks 


\section{Discussion}

- Adaptive behavior

- Speed increase but not significant with cellphone use under dense traffic

- Few cellphone use events under dense traffic, drivers might avoid to use cellphone under high driving demand situations

- Two participants contributed most of cellphone use events, might be risker drivers

- Maintain traffic flow which might cause increased driving demand

- Further examinations are needed with larger sample size 


\section{Future Work}

- No critical situations occurred in this one week driving duration

- Future analysis could focus on safety critical events, such as crash or near crash events 


\section{Questions?}

Huimin Xiong: xionghm@umich.edu Shan Bao: shanbao@umich.edu Jame Sayer: jimsayer@umich.edu 